\title{
SURVEY OF ANALYSIS AND PERFORMANCE OF OFDM SIGNALS IN TIME AND FREQUENCY DISPERSIVE CHANNELS
}

\author{
Vaibhav Singh ${ }^{1}$, G.S. Tripathi ${ }^{2}$ \\ ${ }^{1}$ Department of Electronics, Madan Mohan Malviya Technical University, Gorakhpur- U.P. (India) \\ ${ }^{2}$ Department of Electronics, Madan Mohan Malviya Technical University, Gorakhpur- U.P. (India)
}

\begin{abstract}
The increase in the number of wireless devices and the requirement for higher data rates places an increasing demand on bandwidth. This necessitates the need for communication systems with increased throughput and capacity. Multiple input multiple output orthogonal frequency division multiplexing (MIMO-OFDM) is one way to meet this need. OFDM is used in many wireless communication devices and offers high spectral efficiency and resilience to multipath channel effects. Though OFDM is sensitive to synchronization errors, it makes the task of channel equalization simple. MIMO makes use of multiple antennas to increase throughput without increasing transmitter power or bandwidth. Several modulation schemes are presented that are often used in conjunction with OFDM . Mathematical definitions and analysis of OFDM are given along with a discrete implementation common to modern communication systems. Synchronization errors are described mathematically, as well as techniques to estimate and correct those errors at the receiver.
\end{abstract}

\section{INTRODUCTION}

The year 2010 saw global mobile data usage increase by almost threefold [4]. This increased data usage is from the rise in the number of consumer electronic devices that rely on wireless standards such as IEEE802.11 (Wi-Fi), IEEE802.16 (WiMAX), and cell phones in particular multifunction smart phones [27]. The rising number of devices that require high data rates is placing increasing demands on bandwidth. One of the ways these challenges are being met is with the use of orthogonal frequency division multiplexing (OFDM). OFDM is not only spectrally efficient but resilient to the effects of the multipath wireless channel.Orthogonal frequency-division multiplexing (OFDM) is widely employed in wireless communication systems like digital audio broadcasting (DAB) [1], digital video broadcasting-terrestrial (DVB-T) [2], and HYPERLAN/ 2 [3] for wireless local-area network (WLAN). The mixture of code-division multiple access (CDMA) and OFDM is considered to be a good candidate for future generation mobile wireless systems [4]. Wireless communications are generally subject to severe multipath fading channels that can seriously degrade the system performance. Counteracting the frequency selectivity of multipath channels by multiplexing information on different orthogonal carriers is the key to the OFDM success. Indeed, if a cyclic prefix is inserted between successive OFDM symbols, the overall system can be viewed as composed of parallel frequency flat channels [4]-[6]. In modern communication systems, which take advantage of the fast Fourier transform (FFT) processing to realize an OFDM modem, the channel induced distortions can then easily be compensated at the receiver side by a complex multiplication of each FFT output. An OFDM signal is generally characterized by the sum of a high number of carriers. This causes a highly variable envelope, which makes the technique sensitive to nonlinear distortions introduced by real hardware (power amplifiers, analog-todigital-digital-to-analog (A/D-D/A) converters, etc.) Another technique being used is multiple input multiple output (MIMO). MIMO has already been incorporated into the IEEE802.11n, IEEE802.16e, and 4G cellular wireless standards. The introduction of MIMO into these standards make possible the increased data throughput and range required by many devices without increases in transmit power or bandwidth.

\section{THE CHANNEL}

The success of any communication system model resides with the accurate representation of the channel. This is especially so with the wireless channel. Effects which are non-existent or negligible in a wired communication system can render a wireless channel unusable unless measures are taken to counteract them. Variations in channel statistics, called propagation mechanisms, can be placed into three categories: small, mid, and large scale effects [10]

\subsection{Large Scale Effects}

Large scale propagation effects are those which occur at large distances, many times the RF carrier wavelength. Two types of large scale propagation effects are path loss and shadowing. Path loss is the simplest propagation mechanism to model as it is the decrease in signal power as a function of distance. Consider the simplest case where the transmitter and receiver are isotropic antennas and are separated by a distance $r$ in a free space environment as shown in Figure 2.1. We assume the antenna radiates isotropically and thus the power is the same along the sphere with the transmit antenna at the origin. It is easy to see that the power will decrease as the reciprocal of the distance squared. The path loss FL can be described, in positive terms, by. 


$$
\mathrm{FL}=\left(\frac{4 \pi r^{2}}{\lambda}\right)
$$

where wavelength $\lambda$, frequency $f$, and the speed of light $\mathrm{c}$ are related by $\lambda=\mathrm{c} / \mathrm{f}$. This model does have is its limitations however. Note that as $\mathrm{r} \rightarrow 0$ the free space loss FL $\rightarrow \infty$ which clearly can not happen since this indicates an increase in power, greater than that transmitted. Figure 2.2 demonstrates this anomaly with $\mathrm{f}=900 \mathrm{MHz}$ and distance in meters. The second large scale propagation model is shadowing. This effect is caused by path obstructions like buildings, vegetation, etc. Though models exist that use information from geographical information systems (GIS) databases, they are used mainly for cell tower placement[16]. Because of the difficulty in accounting for the voluminous causes of shadowing and the distances involved, we will not consider this effect in our model

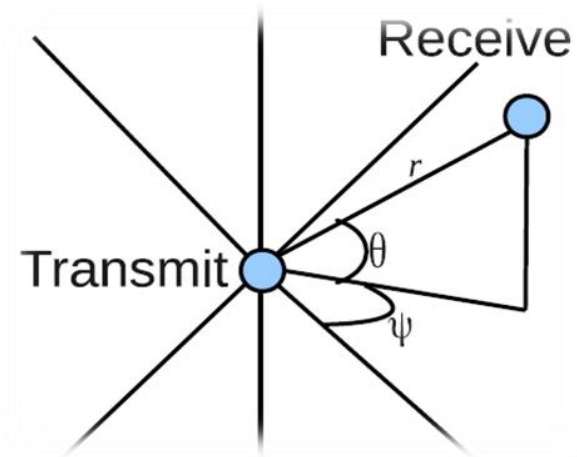

Fig 2.1 Free space model

\subsection{Mid-Scale Effects}

Mid scale effects are variations in the channel for same antenna separation distance and same local area. This effect can be seen when two sets of antennas are in the same room with the same distance but with drastically different attenuations in the different paths. These effects will also be excluded from our model except were they contribute to small scale.

\subsection{Small Scale Effects}

These are the effects that we will devote our attention to and derive models for mostly because for indoor applications they are the dominant effect. The first small scale effect we consider is Doppler shift. This is caused by either the transmitter or the receiver antenna moving but for convenience we assume that the transmit is fixed, without loss of generality. The movement of the antenna will have an effect on the frequency of the transmitted signal. We saw previously that wavelength and frequency are related by the speed of light, $\lambda=\mathrm{f} / \mathrm{c}$, for stationary antennas. The change in frequency or Doppler shift is dependent on the velocity of the antenna, $v$, and the angle between the line of sight and the direction of motion, $\theta$, [26],

$$
\Delta \mathrm{f}=\frac{v}{c} \mathrm{f} \cos \theta
$$

Equation does not take into account relativistic properties such as time dilation and so the equality is an approximation. Figure 2.3 shows the Doppler shift as a function of $\mathrm{v}$ and $\theta$. Note that for $|\theta|=\pi / 2$, there is no Doppler shift which is what we would expect if the receive antenna is moving perpendicular, or transverse, to the transmit antenna. The second small scale effect is multipath fading. This propagation mechanism is the result of the constructive and destructive interference from the multiple paths taken between Tx and Rx antenna. These paths can be the line of sight (LOS) or reflections from structures or terrain. The first graph shows that when the two paths have the same phase the sinusoids add constructively.

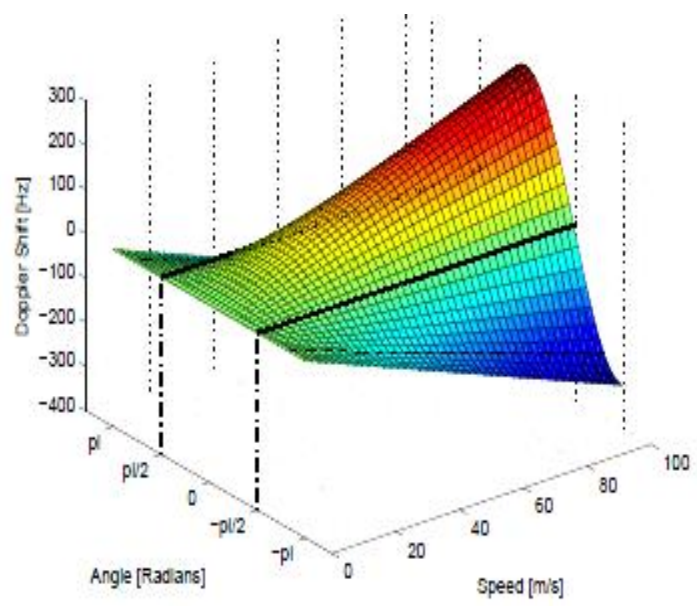

Doppler shift as a function of speed and angle relative to the transmit antenna

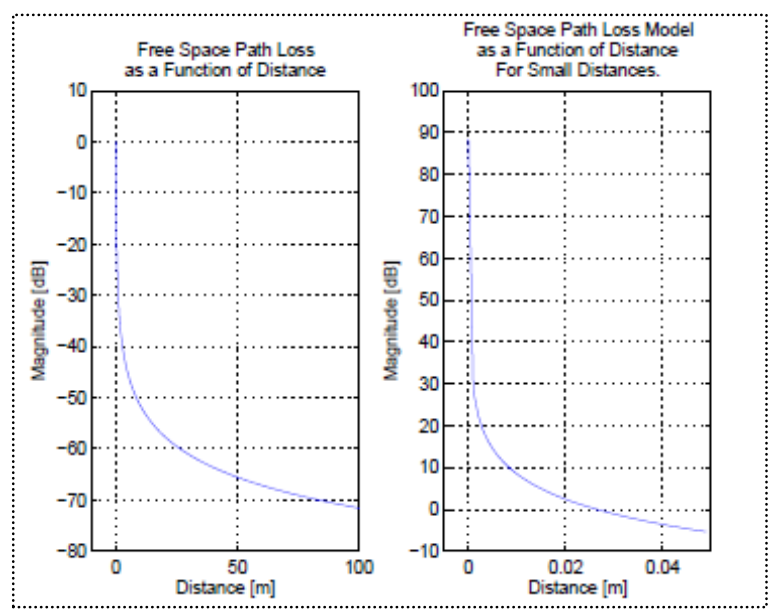

Fig 2.2 Free space path loss model for large and small distances

\section{FREQUENCY DOMAIN CHANNEL MODEL}

In this section we discuss the implementation of a channel model that is derived in the frequency domain. This model presented in [21] with code in Appendix A, is particularly useful for OFDM simulation in small local areas (i.e. indoor applications). We have already looked at the channel parameters in the time domain but we need to redefine them in the frequency domain. 
The first channel characteristic we will consider is the channel correlation function,

$$
\varphi \mathrm{H}(\mathrm{f} 1, \mathrm{f} 2, \mathrm{t} 1, \mathrm{t} 2)=\mathrm{E}\{\mathrm{H} *(\mathrm{f} 1, \mathrm{t} 1) \mathrm{H}(\mathrm{f} 2, \mathrm{t} 2)\}
$$

and since we are assuming that the channel is time invariant for some time interval

$$
\phi \mathrm{H}(\Delta \mathrm{f})=\phi \mathrm{H}(\Delta \mathrm{f}, 0)
$$

We will base the channel model on the delay power spectrum, with delay spread maximum $\tau$ max, which is the Fourier transform of the spaced frequency correlation function with coherence bandwidth $\delta f$. The coherence time is the time duration where the channel impulse response is considered not varying or where the channel transfer function shows significant correlation. The coherence bandwidth is the range of frequencies where thechannel is considered flat or where the channel transfer function shows significant correlation. The usual related correlation value is 0.9 [21]. The delay power spectrum we will use to define the frequency selectivity has four parameters, $\rho 2$ is the normalized power of the dominant path, _ is the normalized power density of the constant part, $\tau 1$ is the duration of the constant level part, and $\gamma$ is the decay exponent of the exponentially decaying part. The equation for the delay power spectrum is

$$
\phi_{h}(\tau)= \begin{cases}0 & \text { if } \tau<0 \\ \rho^{2} \delta(\tau) & \text { if } \tau=0 \\ \Pi & \text { if } 0<\tau \leq \tau_{1} \\ \Pi e^{-\gamma\left(\tau-\tau_{1}\right)} & \text { if } \tau>\tau_{1}\end{cases}
$$

Figure 2.12 shows the general case for the desired delay power spectrum with constant level part and exponentially decaying part

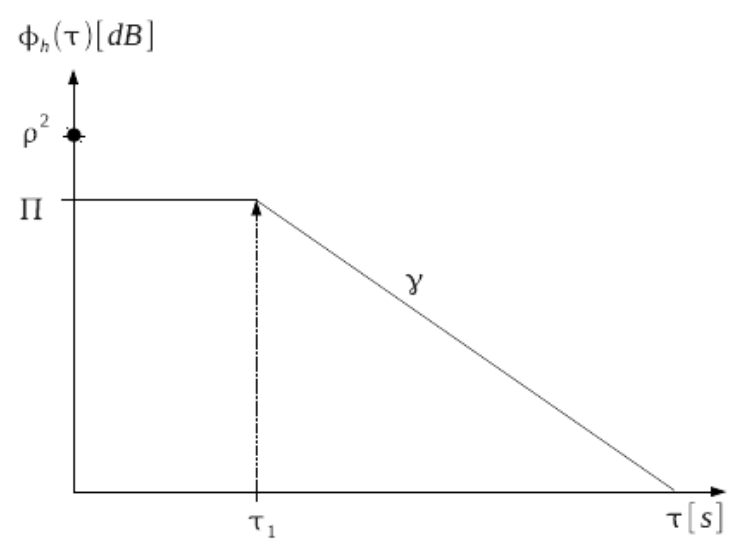

Fig 2.12 Delay power spectrum

For most channels, a good approximation of the channel can be obtained by setting $\tau 1=0$, giving an exponentially decaying delay power spectrum. Our model will contain a dominant LOS path so the mean of the channel transfer function is not zero. We now need to relate the parameters of the delay power spectrum $\{\rho 2,, \gamma, \tau 1\}$ to the channel parameters, namely the normalized received power, $\mathrm{P} 0$, the Rician-K factor, $\mathrm{K}$, and the RDS, $\tau \mathrm{rms}$. The normalized received power $\mathrm{P} 0$ is the sum of the delay power spectrum for all $\tau$ or

$$
\begin{aligned}
p_{0} & =\lim _{m \rightarrow 0} \int_{0}^{m} \phi_{h(\tau)} d \tau \\
& =\lim _{m \rightarrow 0} \int_{0}^{m} \rho^{2} \sigma(\tau) d \tau+\lim _{m \rightarrow 0} \int_{0}^{m} \Pi d \tau e^{-\gamma \tau} \\
& =\rho^{2}+\frac{\Pi}{\gamma}
\end{aligned}
$$

The Rician K-factor is the ratio of the dominant path to the scattered path and so

$$
\begin{aligned}
\mathrm{K} & =\frac{\rho^{2}}{\mathrm{P} 0-\rho^{2}} \\
& =\frac{\rho^{2} \gamma}{\Pi}
\end{aligned}
$$

Table 2.1 lists the parameters of the channel and the model and their relationship.

Table 2.1 Relationship Between Channel Parameters and Model Parameters

\begin{tabular}{|l|l|}
\hline Channel Parameters known & Model Parameters known $\left(\tau_{1}=0\right)$ \\
\hline
\end{tabular}

\begin{tabular}{|c|c|}
\hline \hline$\rho^{2}=P_{0} \frac{K}{K+1}$ & $P_{0}=\rho^{2}+\frac{I I}{\gamma}$ \\
\hline$\gamma=\frac{\sqrt{2 K+1}}{\tau_{\text {rms }}(K+1)}$ & $K=\frac{\rho^{2} \gamma}{\Pi}$ \\
\hline$\Pi=\frac{P_{0}}{K+1} \gamma$ & $\tau_{\text {rms }}=\frac{\sqrt{2 K+1}}{\gamma(K+1)}$ \\
\hline
\end{tabular}

Our model uses an exponentially decaying delay power spectrum and as such has an infinite domain thus we need a maximum excess delay. Though any $\tau$ max can be used, we will set the maximum where the attenuation has decreased by $43 \mathrm{~dB}$ [21]. The maximum excess delay is then $\tau \max =$ $10 / \gamma$ (for the case when $\tau 16=0, \tau \max =\tau 1+10 / \gamma$ ).

In terms of the channel parameters we have

$$
\begin{gathered}
\tau \max =\frac{10}{\gamma} \\
==\frac{10 \tau \mathrm{rms}(\mathrm{k}+1)}{\sqrt{2 k+1}}
\end{gathered}
$$

\section{IMPLEMENTATION OF CHANNEL MODEL}

The path amplitude distribution depends on the presence of a LOS path. For this we will assume that the LOS component is present and therefore use the Rician model. This model assumes that in addition to the LOS path there are a large number of independent smaller (in magnitude) paths. We now look at the implementation of the frequency domain model to obtain the channel impulse response. The process, shown in Figure 2.13, starts by generating real valued noise, passing it through a noise shaping filter, and then using a Hilbert transform (see Section 3.1.4) to make it 
causal and thus complex. Lastly, the LOS component is added in.

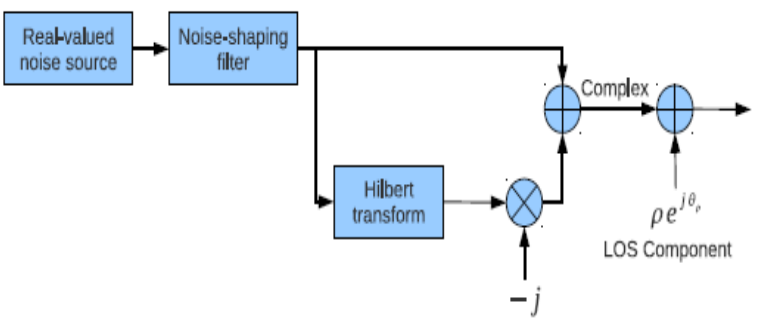

Fig 2.13 The frequency domain model.

The noise shaping filter needs to have a transfer function, $\mathrm{G}(\tau)$, similar to the delay power spectrum. In general, the noise shaping filter transfer function is defined by

$$
|G(\tau)|= \begin{cases}1 & \text { if }|\tau| \leq \tau_{1} \\ e^{-\gamma\left(|\tau|-\tau_{1}\right)} & \text { if }|\tau|>\tau_{1}\end{cases}
$$

but since we have $\tau 1=0$,

$$
|\mathrm{G}(\tau)|=e^{-\gamma|\tau|}
$$

The noise source has variance $\sigma 2$ and the sampled noise has power spectral density (PSD)

$$
\mathrm{S}(\tau)=\sigma^{2} \Delta \mathrm{f}
$$

where $\Delta \mathrm{f}$ is the sampled interval (note that $\Delta \mathrm{f}$ must satisfy the sampling theorem with respect to $\tau \max$ ). The PSD at the output of the noise shaping filter is

$$
s_{g}(\tau)=\sigma^{2} \Delta \mathrm{f}|\mathrm{G}(\tau)|
$$

After adding the Hilbert transform, the PSD increases by a factor of four,

$$
\begin{aligned}
s_{h}(\tau) & =4 \sigma^{2} \Delta \mathrm{f}|\mathrm{G}(\tau)| \\
& =\phi_{h}
\end{aligned}
$$

is Because $\mathrm{G}(\tau)$ is proportional to $\varphi \mathrm{h}(\tau)$ by design, the variance of the noise source is

$$
\sigma^{2}=\frac{\prod}{4 \Delta f}
$$

To test the validity of this model we will use channel parameters obtained from measurements in [13]. These parameters are $\mathrm{P} 0=62.1 \mathrm{~dB}, \mathrm{~K}=1.9 \mathrm{~dB}, \tau \mathrm{rms}=9.0 \mathrm{~ns}, \tau 1=$ 0 , and TF Length $=801$.

Figures 2.14 and 2.15 show the desired delay power spectrum with the noise shaping filter before and after the
Hilbert transform. Recall that we want to simulate a causal channel.

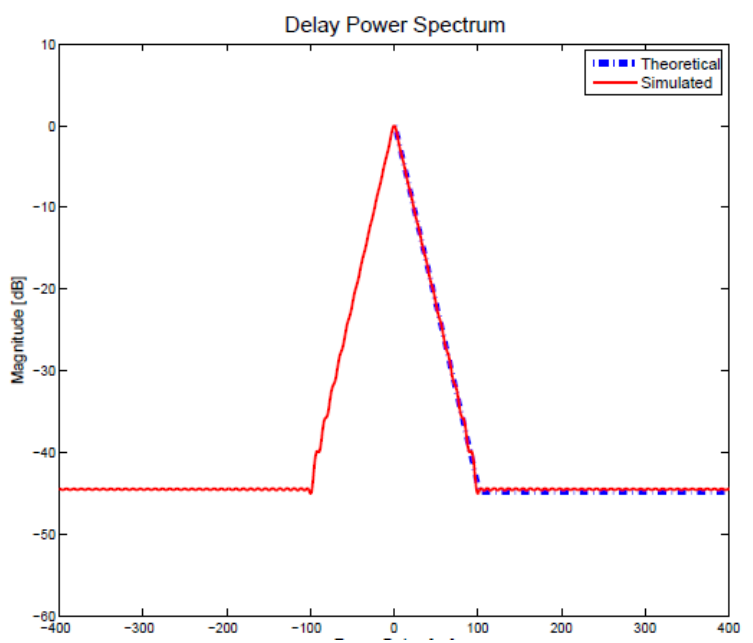

Fig 2.14 Output of noise shaping filter

\section{SYNCHRONIZATION ERRORS AND THEIR ESTIMATIONS}

The most challenging aspect of designing a communication system is the estimation and correction of synchronization errors that occur during transmission. Synchronization of the system in time and frequency is still the source of much research and yet is one of the most often overlooked subjects in published papers [8].

Figure 5.1 shows the block diagram of the synchronization process of a typical receiver. Simulations of the error estimations are presented using the IEEE802.11 standard for the OFDM frame structure

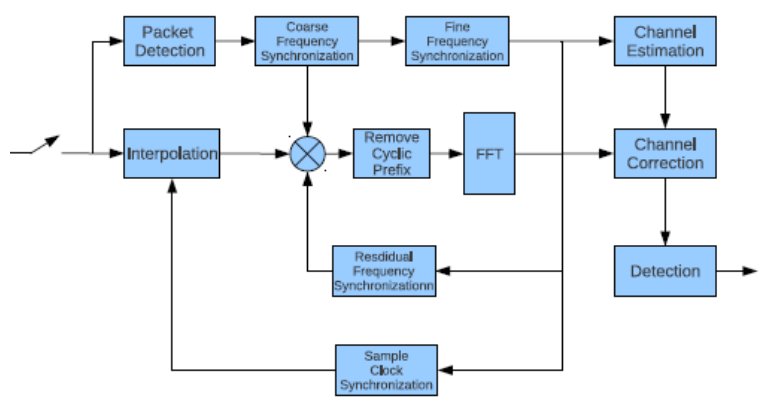

Fig 5.1 Synchronization blocks in OFDM receiver.

\subsection{Synchronization Errors}

As stated earlier, OFDM systems are very sensitive to frequency offsets between transmitter and receiver when modulating to passband and back to baseband. Both ISI and inter channel interference (ICI) need to be mitigated as much as possible in order for the system to accurately receive data.

Much of the ISI can be eliminated with the addition of a guard interval or cyclic prefix. ICI is affected by the orthogonality of the subcarriers, which can be caused by Doppler shift or the offset in carrier frequency between the 
transmitter and receiver. Not only must frequency offsets be dealt with but also the sample clock and the frame or FFT window timing. Offsets in timing can cause ISI as well as ICI

\subsubsection{Sampling Clock Offset}

At the receiver the incoming signal is sampled with an analog to digital converter (ADC). The ADC is driven by the receiver clock which, in practice, is not perfectly

Effects of Sample Clock Offset on Spectrum

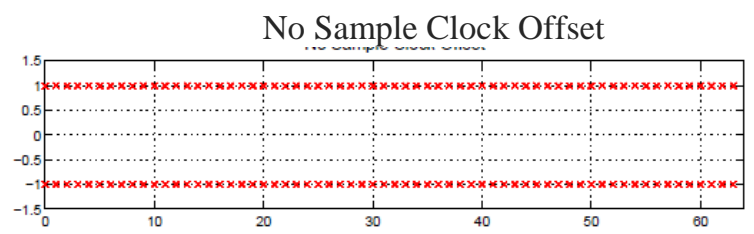

Sample Clock Offset of $1 \%$ With No ICI

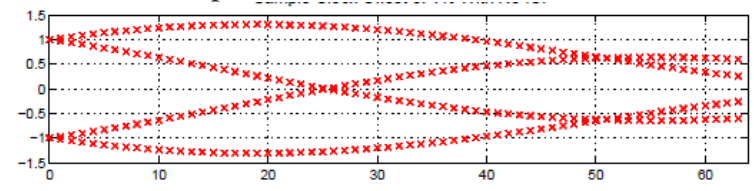

Sample Clock Offset of $1 \%$ With ICI

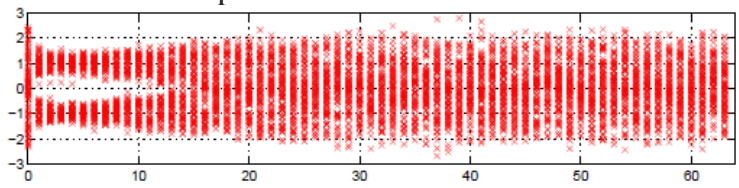

Effects of sample clock offset on spectrum

\subsubsection{Frame Timing Offset}

The estimation of the OFDM symbol or frame start position determines the alignment of the FFT window with the noncyclically extended OFDM symbol. An offset in the FFT window can then include a neighboring OFDM symbol causing ISI, which can affect the orthogonality of the subcarriers producing ICI. Analysis of the effects of frame timing offset on the constellation and the spectrum will be discussed with and without the use of a cyclic prefix for QPSK. We proceed first with the latter case.

Consider the frame start positions as presented in Figure 5.16 where there is the addition of a cyclic prefix. There are two possible cases for frame start regions. If the start point is within region $\mathrm{A}$, it is unaffected by the multipath channel and the FFT window is within the correct OFDM symbol. The addition of the cyclic prefix extends the OFDM symbol allowing for the offset FFT window to remain within the correct OFDM symbol. If the start position is within region $\mathrm{B}$, it will either be affected by the multiple paths (an early start) or extend into the next OFDM symbol causing ISI and ICI.

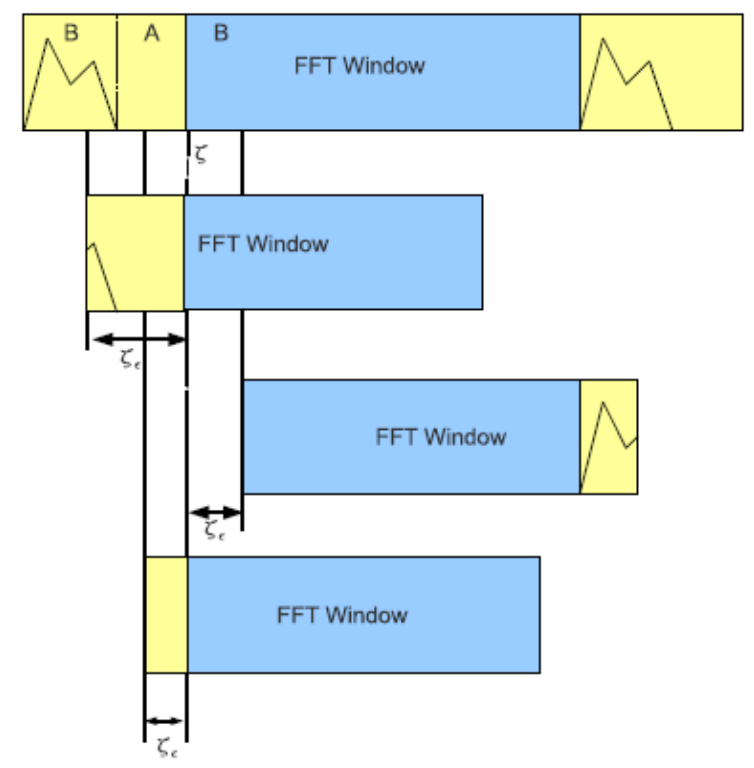

Fig 5.16 Possible locations for frame start position.

\section{Frequency Offset -}

Let _fc $[\mathrm{Hz}]$ be the carrier frequency offset and the normalized carrier frequency offset, $\mathrm{Q}$, be

$$
\varepsilon=\frac{\Delta f c}{\Delta f}
$$

where $\Delta \mathrm{f}$ is the sub-carrier bandwidth (sub-carrier spacing). There is effect of normalized frequency offset on inter channel interference. If normalized frequency offset increase then power of inter channel interference also increase.It can be seen from the below graph figure 5.6.

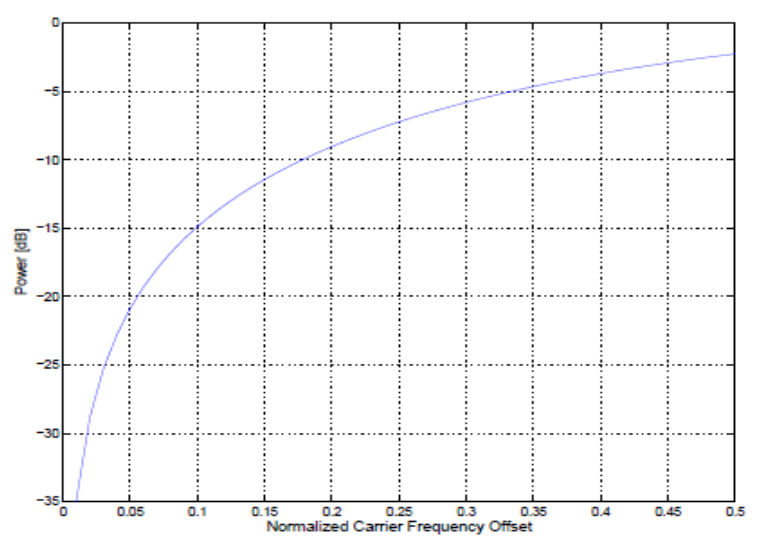

\subsection{Synchronization Error Estimation}

Synchronization is separated into two categories, acquisition and tracking. In acquisition we focus on carrier frequency offset estimation, symbol timing and frame start position using the preamble structure outlined in IEEE802.11a [12]. Tracking occurs after the preamble has been sent and relies on information embedded into each OFDM frame to ensure carrier frequency and sample clock are locked during transmission 


\subsubsection{Preamble Structure of IEEE802.11a -}

Because of the sensitivity that OFDM has to frequency and timing offsets, measurements must be taken to estimate and correct these offsets at the receiver. To accomplish this and other signal processing functions, four OFDM symbols are prepended to the OFDM burst transmission to aid in acquisition. After this preamble is processed, each successive frame contains four pilot symbols that are used for tracking frequency and timing. Both the symbols in the preamble and the pilot symbols are known to the receiver. The first two OFDM frames contain ten short preambles and are used for automatic gain control, diversity selection, timing acquisition, and coarse frequency acquisition. We will focus our attention on the acquisition of timing and frequency.

\subsubsection{Frequency Offset Estimation}

Estimation of frequency offset during the acquisition phase is performed in two parts. Coarse frequency offset uses the short training symbols and fine frequency offset uses the long training symbols. Both coarse and fine frequency estimation use the same algorithm, correlation with the received signal and a delayed copy. The conjugate product is then passed to a phase detector that outputs the phase error.

\subsubsection{Frequency Estimation Algorithm}

Short training symbols are used for the coarse estimation and correction before using the long training symbols for fine frequency estimation and correction. Figure 5.33 shows the frequency estimation algorithm in which we use the corrector.

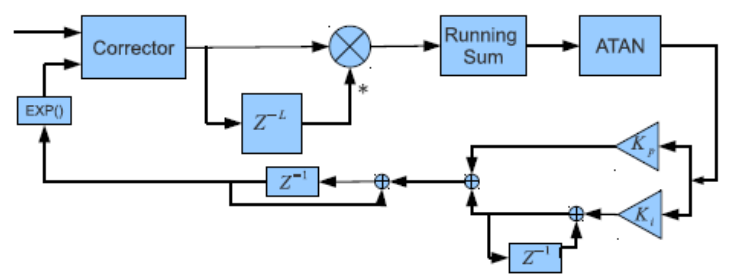

Frequency estimation algorithm

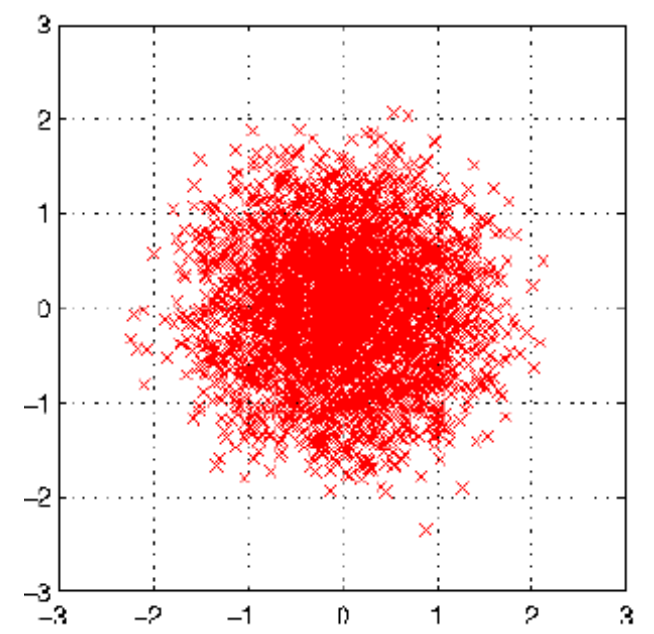

Constellation without frequency offset correction

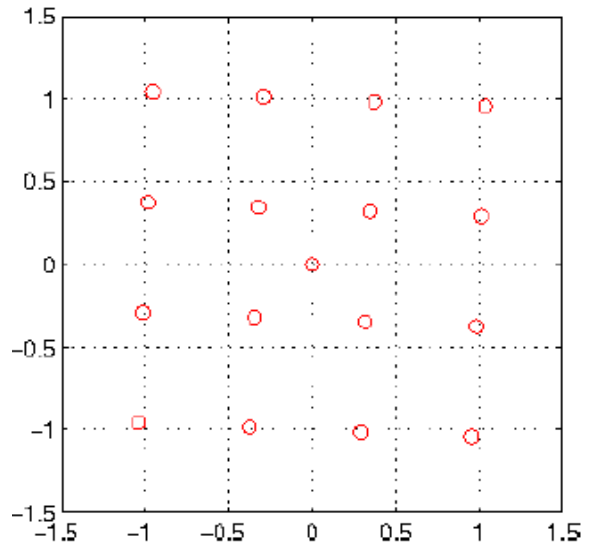

Constellation with frequency offset correction.

\section{CONCLUSIONS}

This paper presented an introduction to OFDM, specifically, the topics of the channel, OFDM, synchronization anddetailed the effects present in a wireless multipath channel and the importance of the channel model. To that end, a frequency domain channel model was given for the wireless multipath fading channel. How the synchronization errors could be estimated at the receiver and corrected

This paper aimed to be an introductory look into OFDM. Future systems will include MIMO-OFDM and, as such, there is a lot of research being done in this area. One of the assumptions made about the channel was that it was static. While this assumption works well for standards such as IEEE802.11, it does not work well for cell phones and other wireless devices. In the future, we may see many devices that must be able to operate at speeds were Doppler shifts affect data transmission.

\section{REFERENCES}

[1]. S. ALAMOUTI, A simple transmit diversity technique for wireless communications, IEEE Journal on Selected Areas in Communications, 16 (1998), pp. $1451-1458$.

[2]. A. ANTONIOU, Digital Signal Processing, McGrawHill, New York, New York, 2006.

[3]. R. CHANG, Synthesis of band-limited orthogonal signals for multi-channel data transmission, Bell System Technical Journal, 46 (1966), pp. 1775-1796.

[4]. F. J. HARRIS, Orthogonal frequency division multiplexing. Unpublished notes, 2010

[5]. H. HASHEMI, The indoor radio propagation channel, Proceedings of the IEEE, 81(7) (1993), pp. $943-968$.

[6]. H. NIKOOKAR AND H. HASHEMI, Phase modeling of indoor radio propagation

[7]. L. J. Cimini Jr., "Analysis and simulation of a digital mobile channel using orthogonal frequency division multiplexing," IEEE Trans. Commun., vol. 33, pp. 665-675, July 1985.

[8]. P. Banelli and S. Cacopardi, "Theoretical analysis and performance of OFDM signals in nonlinear AWGN channels," IEEE Trans. Commun., vol. 48, pp. 430-441, Mar. 2000. 